Article

\title{
Catalytic activity of palladium nanoparticles immobilized on an amino-functionalized ceramic membrane support
}

\author{
Hong Jiang a , Xiaoxu Sun a, Yan Du b, Rizhi Chen a,*, Weihong Xing a,\# \\ a State Key Laboratory of Materials-Oriented Chemical Engineering, Nanjing Tech University, Nanjing 210009, Jiangsu, China \\ ${ }^{\mathrm{b}}$ College of Environment, Nanjing Tech University, Nanjing 210009, Jiangsu, China
}

\section{A R T I C L E I N F O}

Article history:

Received 22 May 2014

Accepted 3 July 2014

Published 20 December 2014

\section{Keywords:}

Palladium nanoparticle

$N$-( $\beta$-aminoethyl)- $\gamma$-aminopropyl

trimethoxy silane

Ceramic membrane

$p$-Nitrophenol hydrogenation

\begin{abstract}
A B S T R A C T
Pd nanoparticles were immobilized on a tubular ceramic membrane support. The support surface was functionalized by $N$-( $\beta$-aminoethyl)- $\gamma$-aminopropyl trimethoxy silane (AAPTS), which contains two amino groups. The Pd-immobilized ceramic membrane support was characterized by X-ray diffraction, field-emission scanning electron microscopy, energy-dispersive X-ray spectroscopy, inductively coupled plasma emission spectroscopy, X-ray photoelectron spectroscopy, and high-resolution transmission electron microscopy. Its catalytic properties were investigated by the liquid phase hydrogenation of $p$-nitrophenol to $p$-aminophenol. The Pd-immobilized ceramic membrane support was compared with the Pd nanoparticles immobilized on a similar support functionalized by $\gamma$-amino-propyltriethoxy silane (3-APTS), which contains one amino group. Higher catalytic activity and stability were observed for the AAPTS-functionalized support. AAPTS contains twice as many amino groups as 3-APTS, and consequently exhibited a stronger electron-donating effect toward Pd. The AAPTS-functionalized ceramic membrane support contained more immobilized Pd nanoparticles, which were bound more strongly. This led to a higher catalytic activity and stability.
\end{abstract}

(C) 2014, Dalian Institute of Chemical Physics, Chinese Academy of Sciences. Published by Elsevier B.V. All rights reserved.

\section{Introduction}

Metal nanoparticles exhibit unique electronic, optical, and catalytic properties because of the quantum size effect. They have potential in applications including electrochemical immunosensors and catalysts in chemical and photochemical reactions [1-5]. Their large surface-to-volume rations make them attractive in catalysis, and Pt and Pd nanoparticles have received enormous attention in this respect [6]. Stabilized clusters and colloids of Pd with nano-scale dimensions can catalyze organic and inorganic reactions $[7,8]$. However, sepa- rating the $\mathrm{Pd}$ nanoparticles from the reaction products and recyclability without loss of catalytic activity remain significant challenges [9]. Immobilizing catalyst particles on membranes allows the catalyst to be easily separated from the reaction medium and potentially reused without loss of activity [10-13]. $\mathrm{Al}_{2} \mathrm{O}_{3}, \mathrm{TiO}_{2}$, and $\mathrm{ZrO}_{2}$ membranes are attractive catalyst supports because they can withstand high temperatures and/or pressures and high concentrations of corrosive products [14].

The surface properties of the support influence the size and dispersion of the metal particles, as well as the catalytic performance $[15,16]$. Functionalizing supports with chelating lig-

\footnotetext{
* Corresponding author. Tel: +86-25-83172286; Fax: +86-25-83172292; E-mail: rizhichen@njtech.edu.cn

\# Corresponding author. Tel: +86-25-83172288; Fax: +86-25-83172292; E-mail: xingwh@njtech.edu.cn

This work was supported by the National Natural Science Foundation of China $(21106061,21125629)$ and the Natural Science Foundation of Jiangsu Province (BK20130920). 
ands can overcome metal catalyst deterioration and leaching, can stabilize the metal particles, and can increase the catalytic efficiency [17]. Amino groups bind strongly to metal nanoparticles [18], so functionalizing supports with amino groups can increase their loading capacity of metal particles. The improved adhesion between the metal particles and support also leads to superior catalytic properties [19]. $\gamma$-Amino-propyltriethoxy silane (3-APTS) is a common silane coupling agent. We previously functionalized the surface of an alumina tubular membrane support and hollow fiber ceramic membrane support with 3-APTS [20,21]. 3-APTS can react with surface-OH groups through condensation, forming $-\mathrm{Si}-\mathrm{O}-\mathrm{Al}-$ bonds. $-\mathrm{NH}_{2}$ groups are strong electron donors and good ligands for transition metal ions due to their lone electron pair. Pd(II) can coordinate with 3-APTS and become bound to the ceramic membrane (CM) support. This enhances the Pd loading and improves the catalytic properties of the immobilized Pd nanoparticles [20] The microstructure of the amino-functionalized silane, such as its amino group content, affects the loading of metal nanoparticles and corresponding catalytic performance [22].

$N$-( $\beta$-aminoethyl)- $\gamma$-aminopropyl trimethoxy silane (AAPTS) contains two amino groups (Fig. 1). In the present study, the CM support surface was modified with AAPTS to increase the loading of Pd nanoparticles and improve its catalytic properties. Pd nanoparticles were immobilized on the AAPTS-functionalized CM support. The resulting catalyst exhibited enhanced activity in the liquid phase hydrogenation of $p$-nitrophenol to $p$-aminophenol. The microstructure of the Pd-immobilized CM support was characterized by X-ray diffraction (XRD), field-emission scanning electron microscopy (FESEM), energy-dispersive X-ray spectroscopy (EDS), inductively coupled plasma (ICP) emission spectroscopy, X-ray photoelectron spectroscopy (XPS), and high-resolution transmission electron microscopy (HRTEM). The current Pd-immobilized CM support was compared with Pd nanoparticles deposited on a 3-APTS-functionalized CM support, which was the subject of our previous report [20].

\section{Experimental}

\subsection{Catalyst preparation}

A tubular CM support (Nanjing High-Tech Co., Ltd., Nanjing, China) with inner and outer diameters of 8 and $12 \mathrm{~mm}$, respectively, and length of $60 \mathrm{~mm}$ was used as the starting material. The CM support consisted of a thin $\alpha-\mathrm{Al}_{2} \mathrm{O}_{3}$ layer (nominal pore size of $0.2 \mu \mathrm{m}$ ) on the outer wall of an $\alpha-\mathrm{Al}_{2} \mathrm{O}_{3}$ porous support (nominal pore size of $3 \mu \mathrm{m}$ ). AAPTS (Nanjing Capatue Chemical Co., Ltd., Nanjing, China) was used as the silane coupling agent.

The immobilization of $\mathrm{Pd}$ nanoparticles on the amino-functionalized CM support is shown in Fig. 1. The tubular CM support was sealed at each end and immersed in $50 \mathrm{ml}$ of $0.2 \mathrm{~g} / \mathrm{L}$ silane in dichloromethane at room temperature for $8 \mathrm{~h}$. The CM support was then washed with ethanol to remove unreacted silane and dried at room temperature. The silanized CM support was impregnated with $25 \mathrm{ml}$ of $0.04 \mathrm{~mol} / \mathrm{L}$ aqueous $\mathrm{Pd}(\mathrm{OAc})_{2}$ and kept at $30{ }^{\circ} \mathrm{C}$ for $18 \mathrm{~h}$. The impregnated CM sup-

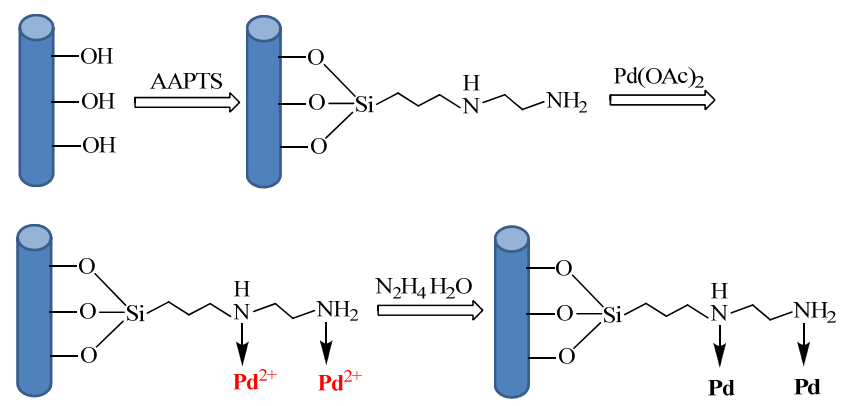

Fig. 1. Schematic representation of the immobilization of Pd nanoparticles on the CM support.

port was then reduced by reaction with $11 \mathrm{~mL}$ of $0.015 \mathrm{~mol} / \mathrm{L}$ aqueous $\mathrm{N}_{2} \mathrm{H}_{4} \cdot \mathrm{H}_{2} \mathrm{O}$ in an ice bath for $30 \mathrm{~min}$. The CM support surface blackened during reduction, indicating the formation of metallic Pd nanoparticles. The resulting Pd-immobilized CM support (Pd-AAPTS-CM) was rinsed thoroughly with distilled water and dried at room temperature.

\subsection{Catalyst characterization}

The Pd content was determined by ICP emission spectroscopy using an Optima 2000 DV system (PerkinElmer, USA). Samples were first digested in $10 \%(v / v) \mathrm{HNO}_{3}$ at $60{ }^{\circ} \mathrm{C}$ for $1 \mathrm{~h}$. Powder XRD patterns were acquired on a Dmax/RB instrument (Rigaku, Japan) using Ni-filtered $\mathrm{Cu} K_{\alpha}$ radiation ( $\lambda=0.15418$ $\mathrm{nm}$ ) at $40 \mathrm{kV}$ and $40 \mathrm{~mA}$ from $2 \theta=30^{\circ}$ to $70^{\circ}$ at a scanning rate of $0.5^{\circ} \mathrm{s}$. The surface morphology was observed by FESEM (Hitachi S-4800, Japan), and the composition was determined by EDS (NORAN System Six, USA). TEM images were collected on a JEOL JEM-2100 instrument (Japan) operated at $200 \mathrm{kV}$. XPS spectra were recorded on a ULVAC PHI 5000 VersaProbe system (Japan) with monochromatic $\mathrm{Al} K_{\alpha}$ radiation (1486.8 $\mathrm{eV}$ ) at $15 \mathrm{kV}$. The residual pressure inside the chamber was 10-10 mbar. The $\mathrm{C} 1 s$ signal at $284.6 \mathrm{eV}$ was used to calibrate binding energies.

\subsection{Catalytic properties}

The catalytic properties of the Pd-AAPTS-CM support were investigated by the catalytic hydrogenation of $p$-nitrophenol to $p$-aminophenol (Scheme 1) in a $300 \mathrm{~mL}$ stainless steel autoclave.

In a typical reaction, $14 \mathrm{~g}$ of $p$-nitrophenol dissolved in 163 $\mathrm{mL}$ of ethanol was loaded into the reactor, and the Pd-AAPTS-CM support was fixed inside the autoclave. The reactor was flushed with hydrogen five times, and heating was commenced under slow stirring. When the desired tempera-

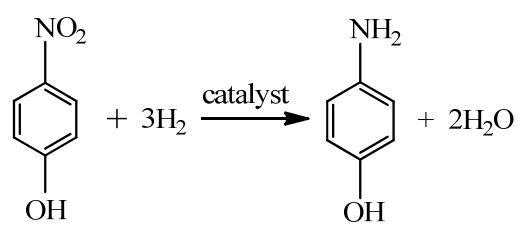

Scheme 1. Catalytic hydrogenation of $p$-nitrophenol to $p$-aminophenol. 
ture $\left(102{ }^{\circ} \mathrm{C}\right)$ was reached, the $\mathrm{H}_{2}$ pressure was increased to $1.65 \mathrm{MPa}$, and stirring was increased to $250 \mathrm{rpm}$, thus beginning the reaction. For recycling studies, the Pd-AAPTS-CM support was removed from the reactor, thoroughly washed with ethanol, and dried at room temperature for the next reaction. The reaction was stopped after $1 \mathrm{~h}$. The hydrogenation products were analyzed by high-performance liquid chromatography (HPLC, Agilent 1200 series, USA) equipped with a diode array detector and an autosampler. Chromatographic separations were performed at $35{ }^{\circ} \mathrm{C}$ using a ZORBAX Eclipse XDB-C18 column ( $5 \mu \mathrm{m}, 4.6 \mathrm{~mm} \times 250 \mathrm{~mm})$. The mobile phase was $80 \%$ methanol and $20 \%$ water at a flow rate of $1 \mathrm{ml} / \mathrm{min}$. The hydrogenation rate was expressed by the amount of hydrogen consumed per hour and per surface area of membrane support [20].

\section{Results and discussion}

\subsection{Characterization of the Pd-AAPTS-CM support}

Figure 2 shows XRD patterns of the CM and Pd-AAPTS-CM supports. The broad diffraction peak at $\sim 40^{\circ}$ in the pattern of the Pd-AAPTS-CM support corresponded well with that reported for face-centered cubic (fcc) Pd [23]. Thus, Pd was formed on the CM support. The broadening of the Bragg reflections for Pd on the Pd-AAPTS-CM support indicated that the Pd particles were nanocrystalline.

FESEM images of the CM and Pd-AAPTS-CM supports are shown in Fig. 3. The two samples exhibited obvious differences in surface morphology. White spots contrasting to the grey support were observed on the surface of the Pd-AAPTS-CM support (Fig. 3(b)), which were attributed to immobilized Pd nanoparticles. Elements of higher atomic number typically produce more electrons and appear brighter in the image. Thus, Pd showed up as brighter spots because it has a higher atomic number than $\mathrm{Al}$ and $\mathrm{O}$ [24]. Figure 3(b) shows that the Pd nanoparticles were uniformly dispersed on the CM surface, similarly those deposited on 3-APTS-funtionalized CM support

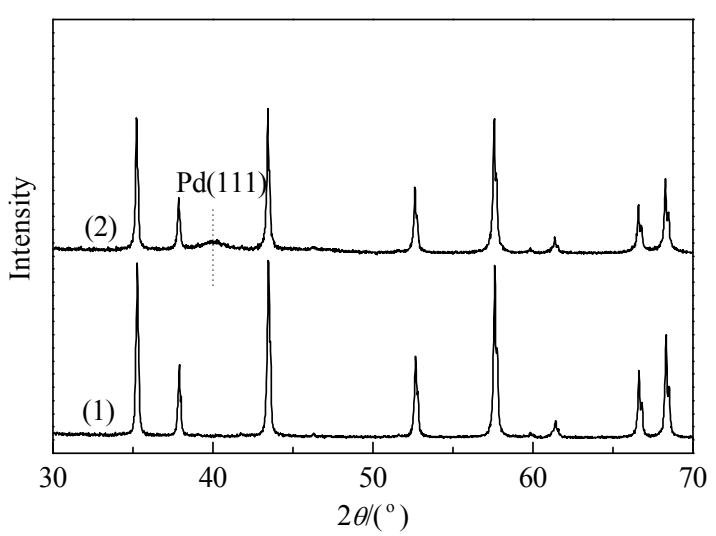

Fig. 2. XRD patterns of the CM (1) and Pd-AAPTS-CM (2) supports.

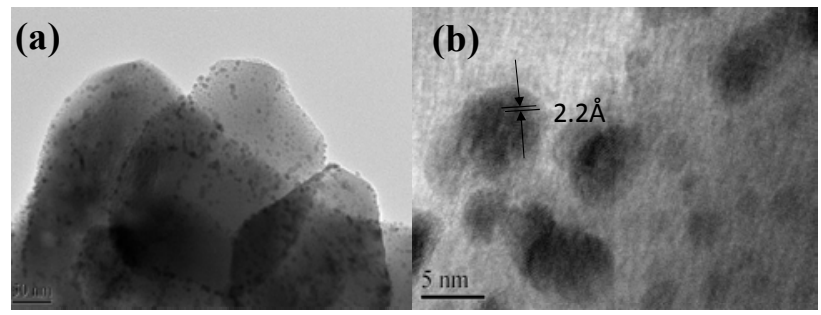

Fig. 4. TEM (a) and HRTEM (b) images of powder recovered from the top membrane layer of the Pd-AAPTS-CM support.

[20]. This indicates that the amino-functionalized silane microstructure had little influence on the dispersion of the Pd nanoparticles. The EDS analysis of a cross section of the Pd-AAPTS-CM support is shown in Fig. 3(c). Pd nanoparticles (white dots) were predominantly located on the surface of the CM support because of its higher area relative compared to the pore support layer [25].

TEM was used to further examine the morphology and particle size of the Pd nanoparticles immobilized on the CM support. Figure 4(a) shows the Pd nanoparticles as dark dots because their electron density is higher than that of $\mathrm{Al}_{2} \mathrm{O}_{3}$. The $\mathrm{Pd}$ nanoparticles were homogeneously immobilized on the CM

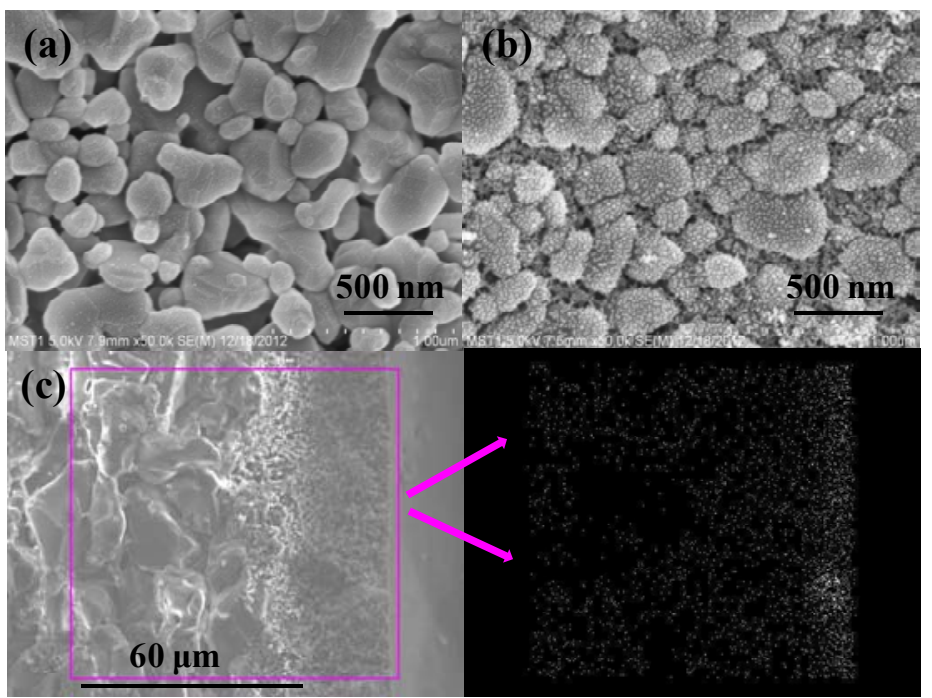

Fig. 3. FESEM images of the surfaces of CM (a) and Pd-AAPTS-CM (b) supports, and EDS analysis (c) of a cross section of the Pd-AAPTS-CM support. 


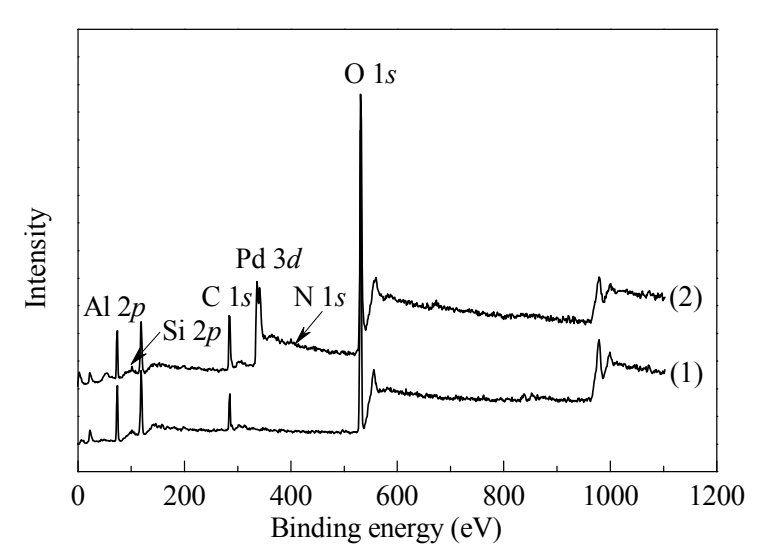

Fig. 5. XPS survey spectra of powders recovered from the top membrane layers of the CM (1) and Pd-AAPTS-CM (2) supports.

support. The HRTEM image (Fig. 4(b)) shows that the mean Pd nanoparticle diameter was $\sim 4 \mathrm{~nm}$, similarly to our previous study [20]. The lattice fringe spacing of $2.2 \AA$ was characteristic of the (111) planes of fcc $\mathrm{Pd}^{0}$ [26].

XPS was used to investigate the chemical states of Pd and N in the Pd-AAPTS-CM support. Figure 5 shows the XPS spectra of powders recovered from the top membrane layers of the CM and Pd-AAPTS-CM supports. Extra peaks were observed in the Pd-AAPTS-CM support. Those at $\sim 400 \mathrm{eV}$ were attributed to $\mathrm{N}$ in amino groups of AAPTS, and those at $\sim 336 \mathrm{eV}$ to Pd. Si $2 p$ peaks were observed for both samples, however, the content of $\mathrm{Si}$ in the Pd-AAPTS-CM support (2.3 at\%) was higher than that in the CM support (1.6 at\%). This indicated the grafting of AAPTS and Pd nanoparticles onto the CM support. Figure 6(a) shows the XPS spectrum of the $\mathrm{Pd} 3 d$ region for the Pd-AAPTS-CM support. A spinorbit doublet was observed, with components at 335.5 and $340.7 \mathrm{eV}$ corresponding to the $\mathrm{Pd}$ $3 d_{5 / 2}$ and $\mathrm{Pd} 3 d_{3 / 2}$ electronic states of $\mathrm{Pd}(0)$, respectively. Peaks at 337.4 and $342.9 \mathrm{eV}$ corresponded to the $\mathrm{Pd} 3 d_{5 / 2}$ and $\mathrm{Pd}$ $3 d_{3 / 2}$ electronic states of Pd(II) species, respectively. This suggested that $\mathrm{Pd}(\mathrm{II})$ was only partially reduced to $\mathrm{Pd}(0)$, in agreement with previous results [20]. The presence of Pd(II) species may have been due to the formation of AAPTS-Pd(II) complexes. Figure 6(b) shows that XPS spectrum of the N $1 s$ region of the Pd-AAPTS-CM support, which was deconvoluted into components at 400.05 and $403.55 \mathrm{eV}$. The higher binding energy signal was attributed to $\mathrm{N}$ in hydrogen-bonded $-\mathrm{NH}_{2}$ and $-\mathrm{NH}-$, and protonated $\mathrm{N}$ in $-\mathrm{NH}_{3}{ }^{+}$and $-\left(\mathrm{NH}_{2}\right)^{+-}$. The lower binding energy signal was attributed to $\mathrm{N}$ in free $-\mathrm{NH}_{2}$ and -NH- groups $[27,28]$. The two N $1 s$ peaks in the Pd-AAPTS-CM support were observed at higher binding energy than those of the aliphatic amino (399.4 eV) and protonated aliphatic amino $(401.5 \mathrm{eV})$ groups in AAPTS. This may have been related to N-Pd coordination [29]. These results confirmed the presence of the silane coupling agent on the CM support surface and chemical bonding of Pd nanoparticles to the CM support surface.

The Pd nanoparticle loading was investigated by ICP emission spectroscopy. The Pd content of the Pd-AAPTS-CM support area $\left(0.43 \mathrm{mg} / \mathrm{cm}^{2}\right)$ was higher than that of the Pd-immobilized
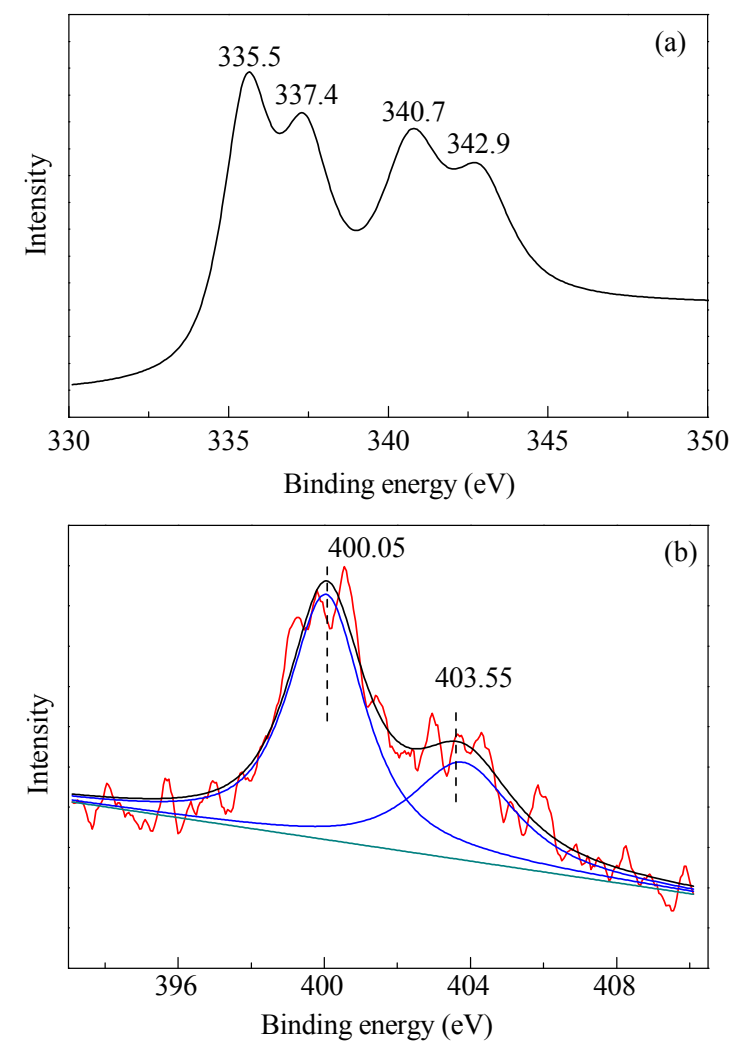

Fig. 6. XPS spectra of the Pd $3 d$ (a) and N $1 s$ (b) regions of powder recovered from the top membrane layer of the Pd-AAPTS-CM support.

3-APTS-funtionalized support $\left(0.32 \mathrm{mg} / \mathrm{cm}^{2}\right)$ [20]. The use of AAPTS with two amino groups increased the Pd nanoparticle loading compared to 3-APTS with one amino group. The Pd nanoparticle loading increased by $34 \%$ compared to our previous work, which was lower than expected. The amino group content of AAPTS is twice that of 3-APTS, so the Pd nanoparticle loading was expected to approximately double. The $\mathrm{N} 1 \mathrm{~s}$ XPS spectrum of the Pd-AAPTS-CM support (Fig. 6(b)) suggested that a fraction of available $-\mathrm{NH}_{2}$ and $-\mathrm{NH}-$ groups became inaccessible to $\mathrm{Pd}$. This was because of ionic bonding between basic amino groups and surface hydroxyl groups [30].

\subsection{Hydrogenation of p-nitrophenol by the Pd-AAPTS-CM support}

Pd and its nanocomposites are well-known catalysts for hydrogenation reactions, carbon-carbon cross-coupling reactions, and the production of $\mathrm{H}_{2} \mathrm{O}_{2}$ from $\mathrm{H}_{2}$ and $\mathrm{O}_{2}$ [31-33]. The catalytic properties of the Pd-AAPTS-CM support were investigated by the hydrogenation of $p$-nitrophenol to $p$-aminophenol in an autoclave. The catalytic activity of the supports was not investigated when acting as membranes, rather as simple catalyst supports [20].

Figure 7 shows the catalytic activity of the Pd-AAPTS-CM support as well as that of the immobilized Pd on the CM support functionalized with 3-APTS (denoted Pd-3-APTS-CM) from our previous study [20]. Apart from the very initial stage, the hydrogenation rate of the Pd-AAPTS-CM support was largely 


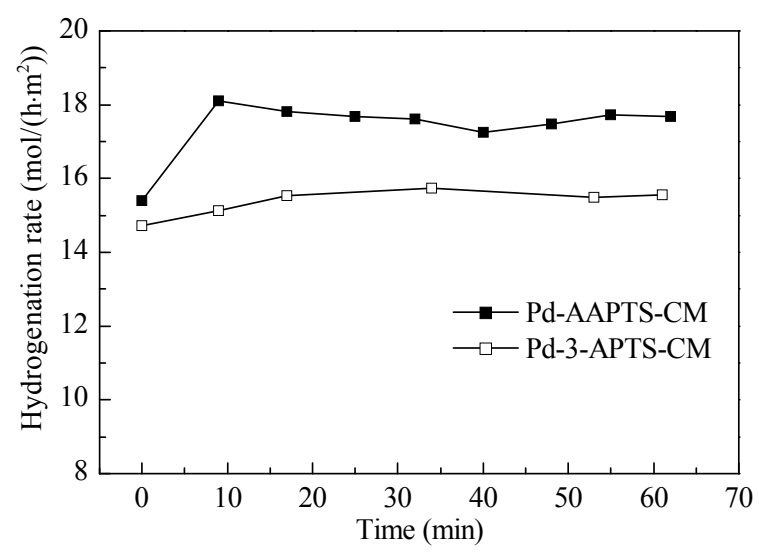

Fig. 7. Variation of $p$-nitrophenol hydrogenation rate with time.

constant at $\sim 17.5 \mathrm{~mol} /\left(\mathrm{h} \cdot \mathrm{m}^{2}\right)$ and was higher than that for the Pd-3-APTS-CM support (15.5 $\mathrm{mol} /\left(\mathrm{h} \cdot \mathrm{m}^{2}\right)$ ) [20]. The increase in hydrogenation rate was not proportional to Pd content because the hydrogenation rate only increased by $13 \%$ (while the Pd content increased by 34\%). This may have been due to the immobilization of Pd nanoparticles on the CM support. This would have resulted in a lower increase in available surface area and catalytic activity, compared to the increase in Pd content. The higher Pd loading was a major factor for the higher catalytic activity of the Pd-AAPTS-CM support. AAPTS contains twice as many amino groups as 3-APTS, so functionalization with AAPTS increased the Pd nanoparticle loading and therefore the catalytic activity.

Consecutive catalytic reactions were carried out to investigate the catalytic stability of the Pd-AAPTS-CM support. The catalytic stability was expressed as the ratio of the hydrogenation rate after a certain number of reactions to that during the first reaction. Figure 8 shows the change in hydrogenation rate with increasing number of catalytic reactions for the Pd-AAPTS-CM support and for the Pd-3-APTS-CM support in our previous study [20]. The catalytic activity of each support decreased until the fourth cycle, and then became constant. After eight consecutive reactions, the Pd-AAPTS-CM support exhibited an $\sim 10 \%$ loss in activity, which was less than that for the Pd-3-APTS-CM support after six reactions (16\%). The cata-

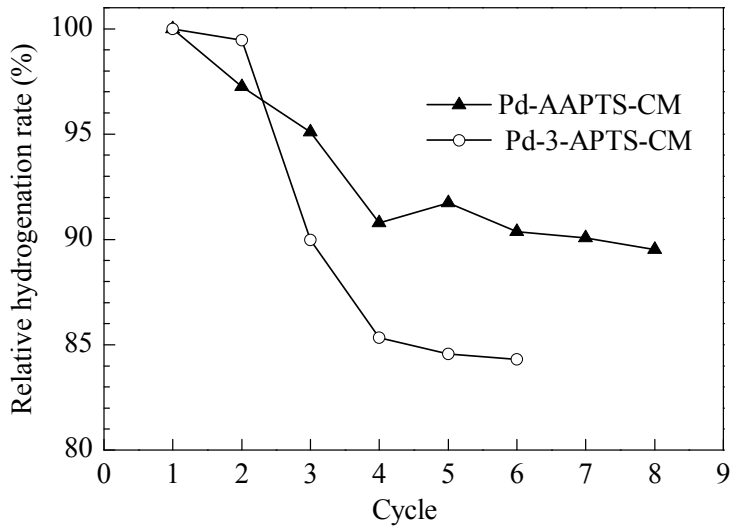

Fig. 8. Catalytic stability of the Pd-AAPTS-CM and Pd-3-APTS-CM supports over consecutive catalytic reactions.

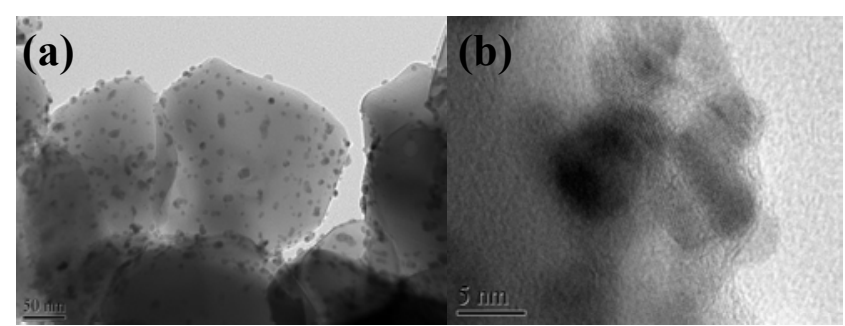

Fig. 9. TEM images of powder recovered from the top membrane layer of the Pd-AAPTS-CM support after eight catalytic reaction cycles.

lytic stability of the Pd-AAPTS-CM support was superior to that of the Pd-3-APTS-CM support. The electron-donating effect of $\mathrm{N}$ toward Pd was greater for the Pd-AAPTS-CM support [34], as verified by the ICP analyses of the used sample. ICP indicated that the Pd content of the Pd-AAPTS-CM support was 0.39 $\mathrm{mg} / \mathrm{cm}^{2}$ of membrane support area after eight consecutive hydrogenation cycles. Thus, the Pd leaching degree was 9\% in the Pd-AAPTS-CM support but $\sim 25 \%$ in the Pd-3-APTS-CM support [20]. ICP also suggested that Pd leaching was one of the main reasons for the deactivation of the Pd-AAPTS-CM support, similar to in our previous study [20,21]. Catalyst deactivation showed a direct correlation with Pd leaching in the current study. TEM images of the Pd-AAPTS-CM support after eight consecutive reactions are shown in Fig. 9. The $\mathrm{Pd}$ dispersion and particle size showed no significant change from the TEM images of unreacted samples (Fig. 4) Thus, the decreased catalytic activity of the Pd-AAPTS-CM support was caused by Pd leaching during hydrogenation reactions.

The hydrogenation products were analyzed by HPLC. Only $p$-aminophenol and $p$-nitrophenol were detected for each support (data not shown). This indicates that the Pd-AAPTS-CM support had high catalytic selectivity in the hydrogenation of p-nitrophenol.

\section{Conclusions}

A CM support surface was modified with APPTS, which contains two amino groups. Pd nanoparticles were then immobilized on the APPTS-functionalized CM support. The resulting Pd-AAPTS-CM support exhibited enhanced catalytic properties, without requiring additional steps to separate the catalyst particles. The Pd-AAPTS-CM support exhibited higher catalytic activity and stability in the hydrogenation of $p$-nitrophenol to $p$-aminophenol, compared to the Pd nanoparticles immobilized on 3-APTS-functionalized CM support. AAPTS contains twice as many amino groups as 3-APTS, and thus exhibited a stronger electron-donating effect toward Pd. Silanes containing high amine functionality can increase the loading of Pd on CM supports and increase their catalytic properties.

\section{References}

[1] Liu N, Chen X, Ma Z F. Biosens Bioelectron, 2013, 48: 33

[2] Lai G S, Zhang H L, Yong J W, Yu A M. Biosens Bioelectron, 2013, 47: 178

[3] Tang B, Tao J L, Xu S P, Wang J F, Hurren C, Xu W Q, Sun L, Wang X 


\section{Graphical Abstract}

Chin. J. Catal., 2014, 35: 1990-1996 doi: 10.1016/S1872-2067(14)60190-X

Catalytic activity of palladium nanoparticles immobilized on an amino-functionalized ceramic membrane support

Hong Jiang, Xiaoxu Sun, Yan Du, Rizhi Chen*, Weihong Xing*

Nanjing Tech University
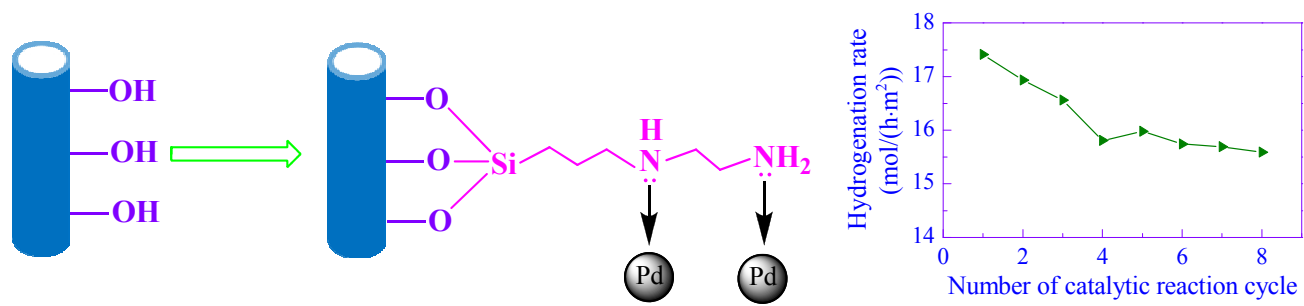

Palladium nanoparticles were deposited on an amino-functionalized tubular ceramic membrane, which exhibited efficient and reusable catalytic activity.

G. Chem Eng J, 2011, 172: 601

[4] Liu R, Yoshida H, Fujita S, Lu N, Tu W H, Arai M. Appl Catal A, 2013, 455: 32

[5] Liang C H, Han J G, Shen K H, Wang L G, Zhao D F, Freeman H S. Chem Eng J, 2010, 165: 709

[6] Mandal S, Roy D, Chaudhari R V, Sastry M. Chem Mater, 2004, 16: 3714

[7] Dablemont C, Lang P, Mangeney C, Piquemal J Y, Petkov V, Herbst F, Viau G. Langmuir, 2008, 24: 5832

[8] Ikeda S, Ishino S, Harada T, Okamoto N, Sakata T, Mori H, Kuwabata S, Torimoto T, Matsumura M. Angew Chem Int Ed, 2006, 45: 7063

[9] Amali A J, Rana R K. Green Chem, 2009, 11: 1781

[10] Domènech B, Mũnoz M, Muraviev D N, Macanás J. Catal Today, 2012, 193: 158

[11] Mertens P G N, Vandezande P, Ye X, Poelman H, De Vos D E, Vankelecom I F J. Adv Synth Catal, 2008, 350: 1241

[12] Volkov V V, Lebedeva V I, Petrova I V, Bobyl A V, Konnikov S G, Roldughin V I, van Erkel J, Tereshchenko G F. Adv Colloid Interface Sci, 2011, 164: 144

[13] Lasri J, Mac Leod T C 0, Pombeiro A J L. Appl Catal A, 2011, 397: 94

[14] Yacou C, Ayral A, Giroir-Fendler A, Baylet A, Julbe A. Catal Today, 2010, 156: 216

[15] Liu C, Tan R, Yu N Y, Yin D H. Microporous Mesoporous Mater, 2010, 131: 162

[16] Williams M, Pineda-Vargas C A, Khataibe E V, Bladergroen B J, Nechaev A N, Linkov V M. Appl Surf Sci, 2008, 254: 3211

[17] Jayamurugan G, Umesh C P, Jayaraman N. J Mol Catal A, 2009, 307: 142

\section{氨功能化陶瓷膜支撑体担载钯纳米颗粒及其增强的催化性能}

\author{
姜 红, 孙晓旭 ${ }^{\mathrm{a}}$, 杜 艳 ${ }^{\mathrm{b}}$, 陈日志 ${ }^{\mathrm{a}}{ }^{*}$, 邢卫红 ${ }^{\mathrm{a}}$, \\ a南京工业大学材料化学工程国家重点实验室, 江苏南京210009 \\ b南京工业大学环境学院, 江苏南京210009
}


镜对催化剂进行了物性表征, 并将其用于催化对硝基苯酚加氢制对氨基苯酚反应. 和单氨基硅烷 $\gamma$-氨丙基三乙氧基硅烷(3-APTS) 功能化改性相比, 担载在AAPTS功能化陶瓷膜上的钯纳米颗粒具有更高的催化活性和稳定性. 相比于3-APTS, AAPTS分子中含 有两个氨基, 具有更强的供电子效应, 因此钯纳米颗粒可更多更稳定地负载在AAPTS功能化陶瓷膜上, 从而具有更高的催化活性 和稳定性.

关键词: 钯纳米颗粒; $N-\beta$-氨乙基)- $\gamma$-氨丙基三甲氧基硅烷; 陶瓷膜; 对硝基苯酚加氢

收稿日期: 2014-05-22. 接受日期: 2014-07-03. 出版日期: 2014-12-20.

*通讯联系人. 电话: (025)83172286; 传真: (025)83172292; 电子信箱: rizhichen@njtech.edu.cn

通讯联系人. 电话: (025)83172288; 传真: (025)83172292; 电子信箱: xingwh@njtech.edu.cn

基金来源：国家自然科学基金(21106061, 21125629); 江苏省自然科学基金(BK20130920).

本文的英文电子版由Elsevier出版社在ScienceDirect上出版(http://www.sciencedirect.com/science/journal/18722067). 